\title{
FACTORS PREVENTING THE USE OF A LIGHTWEIGHT DESIGN WORKFLOW THAT IS INSPIRED BY THE HUMAN LOCOMOTIVE SYSTEM
}

\author{
Uttich, Eike; Bartz, Marcel; Bender, Beate \\ Ruhr-University Bochum
}

\begin{abstract}
A workflow for the design process of technological products was derived from a model that describes the interplay of lightweight design principles in the human locomotive system. This workflow is not yet ready to be used. In this paper, starting points for new research with the goal to enable the use of the workflow are discussed. Using the interplay of lightweight design principles of the human body in technical applications is approached because it is claimed that the interplay leads to an additional reduction of mass. This was proven for a technological system in a previous study. This study lead to a workflow to consider the interplay of the principles in the design process of the technological system. In this paper, the essential parts of this workflow are described in an abstract diagram as a calculation workflow. Subsequently, inputs and outputs of the workflow are identified. Then, the calculation workflow is integrated into the process of design. Afterwards, it is discussed that tension chording, which is one of the lightweight design principles, needs further investigation, because the interplay of the principles can only be used if the principles themselves are used.
\end{abstract}

Keywords: Lightweight design, Bio-inspired design / biomimetics, Early design phases

Contact:

Uttich, Eike

Ruhr-University Bochum

Product Development

Germany

uttich@1pe.rub.de

Cite this article: Uttich, E., Bartz, M., Bender, B. (2019) 'Factors Preventing the Use of a Lightweight Design Workflow that is Inspired by the Human Locomotive System', in Proceedings of the 22nd International Conference on Engineering Design (ICED19), Delft, The Netherlands, 5-8 August 2019. DOI:10.1017/dsi.2019.277 


\section{INTRODUCTION}

There is a constant need for creative ideas to remain competitive in the engineering context. With this need comes the demand for new inspirations. There are several ways to get inspired for an engineer. Nature is becoming a more and more popular one (Hill 1997; Nachtigall 2010). Systems in nature are highly specialised and optimised, all fulfilling the minimum-maximum-principle (Hill 1997). That is, they use a minimum of resources under given boundary conditions. Therefore, nature can provide additional input to current developments in terms of energy efficiency and lightweight design. Biomimetic methods can be used for the transfer from nature to technology (Cohen and Reich 2016; Hill 1997).

An important source of inspiration for engineers developing technical lightweight design systems is the human locomotive system. The lightweight design in the human locomotive system is achieved through the coordinated interplay of different lightweight design principles (Benninghoff et al. 2002; Pauwels 1965 ) that interact with each other. Amongst other effects, this interplay of principles is assumed to lead to mass savings (Pauwels 1965; Gößling et al., 2014). This paper is based on the assumption that lightweight design of the human locomotive system is mainly achieved by the interplay of three lightweight design principles according to Pauwels (1965): (1) functional adaptation and remodelling of bone masses along main stress trajectories, (2) minimisation of bending loads by tension chording through muscles as well as (3) bending minimised motion control of the sensomotoric system.

Recent technical implementations of the aforementioned biological lightweight design principles are mostly focused on the implementation of a single principle. In the context of structural optimisation of technical components, one example is the implementation of biological bone remodelling processes by means of the soft-kill option (SKO) (Mattheck 1997, VDI 6224-3 2017). Another example is the use of the principle of tension chording in robotics and other systems to minimise bending (Witte et al., 2000; Klug et al., 2005; Ananthanarayanan et al., 2012). The technical implementation of individual principles to date has been successful and is commonly used to improve the lightweight design properties of technical systems, but this does not take account of the interplay of the lightweight design principles in the biological role model, which may result in the loss of further lightweight design potential.

According to current studies, the application of the interplay of biological lightweight design principles can, in comparison, save further mass and energy than the use of an isolated design principle (Bartz et al., 2018a; Bartz et al., 2017; Bartz et al., 2018b). In the studies mentioned, the application and implementation of the interplay of the lightweight design principles of the human locomotive system is carried out by using a new bioinspired approach, proposed for technical design calculation. In the human locomotive system, the optimisation strategies take place synchronously. In order to map this in a sequential technical design process, an iterative serial approach is developed with the new bioinspired approach. However, this approach has only been developed on an abstract level by now. Due to the kinematic similarities of the human extremities to technical systems, which can be described as an open kinematic chain, the proposed bioinspired approach is limited to these systems (Bartz et al., 2018b). The advantage of the bioinspired approach in terms of mass savings can be demonstrated by applying it to an articulated arm robot (as an example of a kinematic chain) and comparing it with a classically designed articulated arm robot (Bartz et al., 2018a; Bartz 2019).

However, for various reasons, the new bioinspired approach is not yet ready for a specific technical application. Besides the limitation of the approach to specific systems, the investigation and comparison of the masses in exemplary studies, in this case articulated arm robots, were carried out under isolated conditions. One example for an isolated condition is the defined load case used without control properties. Additionally, the workflow described by (Bartz et al., 2018b) is not yet ready to be used for general applications in early design phases. Therefore, the new bioinspired approach represents a fundamental investigation of the potential for the technique to which this paper refers.

The main goal of this paper is to concretise the new bioinspired approach with the long-term goal of being able to use the approach in technical systems, both to enable the determination of technical fields of application and to improve specific systems. Therefore, this paper tries to classify the new bioinspired approach into the product development process according to Pahl/Beitz (Feldhusen and Grote 2013) and to examine where changes of the previous approach are necessary by considering more specific technical requirements. Another aim of this paper is to discuss on how to find special technical fields of application and specific solutions for possible technical implementations. 
Before the classification and discussion, the biological lightweight design of the human locomotive system is explained in the following section of this paper. This is essential to understand the discussion of the models of the interaction of lightweight design principles presented and the discussion on further proceeding.

\section{THE LIGHTWEIGHT DESIGN PRINCIPLES OF THE HUMAN LOCOMOTIVE SYSTEM AND THEIR INTERPLAY IN NATURE AND TECHNOLOGY}

In the human locomotive system, lightweight design is based on the interplay of different principles. One of those is the hierarchical structure of the bone. This term describes the effect that high stiffness on the macroscopic level is achieved by structures on the microscopic level (Fratzl-Zelman et al., 2011). Hierarchical structures in nature are like composite materials in material science. The hierarchical structure of the bone can be associated with material lightweight design. As the focus for this paper lays on system lightweight design, this principle is not covered in more detail. All other aforementioned principles are associated with system lightweight design.

One lightweight design principle affecting the structure is functional adaptation. A comprehensive example for this principle is the ability of the body to adapt muscle strength when exposed to higher respectively lower loads (Benninghoff et al., 2002). Such exposure could be lifting heavier weights in training. In a more general sense, functional adaptation is a term to describe load dependent mass adaptations of human body parts. In terms of bone mass adaptation, it leads to local mass increases in areas of higher stress and mass decreases in areas of lower stress (Ruff et al., 2006; Frost 2003). It is a part of the regeneration processes in the human body, as it is used to describe the replacement of old bone structures by new ones. In technical terms, this principle is similar to structure optimisation (Mattheck 1997). Functional adaptation is transferred to the technical design process by Mattheck (1997) as topology optimisation, called "Soft Kill Option", and forming optimisation, called "Computer Aided Optimisation". The transfer of functional adaptation was done for the growing behaviour of trees, but similar growing behaviour can be observed for bones.

Another lightweight design principle is tension chording (Pauwels 1965; Benninghoff et al., 2002). In Figure 1.I and Figure 1.II, the two main functions of tension chording are illustrated: minimising tension in the load-bearing structure by tension chords and generating motion by the same chords and forces. To flex the arm, only one muscle would be necessary as shown in Figure 1.II. However, there are at least five muscles which could be used for this motion. This variation problem is called muscle redundancy problem: a defined movement of a body part can be obtained by several variants of muscle activity. To know which muscles are activated to what extent, constraints are needed. Pauwels (1965) assumes that the most relevant constraint for muscle activation is minimal bending. Two extreme cases for results of different muscle activity are displayed in Figure 1.II. In Figure 1.II.A), the activation of muscles leads to close to zero bending in the lower arm. At the same time, bending in the upper arm is relatively high. Opposingly, in Figure 1.II.B) the bending in the upper arm is close to zero. However, bending in the lower arm is high in this case. There are several optimal points between those two cases. More constraints are required to choose one of them. Taking the mass of the system as an additional criterion into account can reduce the number of optimal points. As Pauwels (1965) shows, this can lead to shapes of structures that look similar to their biological examples. Chords and similar actuators can be found in construction machines, e.g. cranes and excavators. They were also tested to reduce bending as additional passive elements or for the actuation of robots (Feyerabend 1991; Witte et al., 2000; Klug et al., 2005).

The last to be mentioned lightweight design principle in the human locomotive system is bending minimised motion control by the sensomotoric system (Pauwels 1965). A simple example: A glass can be moved from a point A to a point B in different ways. One way could be to keep the arm extended as much as possible during the motion. Another way is to move the glass from point $\mathrm{A}$ to the body first and then to point $\mathrm{B}$. When taking only the energy level required as a basis for the decision on which path to choose, the second approach is decided on. Similarly, bending can be a criterion. Pauwels (1965) even claims that bending is considered by motion control of the human locomotive system as a major criterion. He explains it with a limping person. According to him, limping is a reaction of the motion control on pathological stresses in a "normal" gait pattern. From a technological perspective, this motion control behaviour can be described as an optimisation with minimal bending in the loadbearing structure as a constraint. Motion control is well-known in fields like robotics (El Khoury et al., 2013; Mombaur et al., 2014; Weber 2017), however without the consideration of bending 
minimisation. The intersection of the three system lightweight design principles takes place via forces and their distribution in the load-bearing structures. This intersection and the resulting mass saving effects are the starting point of the investigations described in the following.

Each individual lightweight design principle described above can lead to mass savings by itself. In addition, it is shown that the optimal lightweight design in the human locomotive system is achieved through the interplay of the principles (Bartz et al., 2018a; Bartz 2019). By using functional adaptation, oversizing is avoided, i.e. there is just enough mass to ensure the stiffness needed to endure the loads on the bone. As tension chording in combination with bending minimised motion control reduces the bending in load-bearing structures, mass can be saved when coupled with functional adaptation (Pauwels 1965). That means that the interplay of principles leads to increased mass savings: Tension chording leads to minimised bending while increasing compressive forces. Bending minimised path control leads to motion paths in which the remaining bending can be minimised by the tension chords. Bone mass is being reduced at lower stress regions and increased in regions where bending cannot be reduced well by the combination of tension chording and motion control.

To illustrate the effect of the combination of tension chording and functional adaptation, a simple beam is shown in Figure 1.III. The beam and the applied load displayed shall, for the example, remain in the displayed position. Additionally, it shall be rotatable around its joint. A classical approach to this is to add a joint engine to the system, which provides the necessary joint torque to keep the beam in the desired position. To simplify the figure, this is represented by fixed support. As mentioned earlier, bending in a structure can be minimised by structure optimisation. For the beam example, one result is displayed in Figure 1.III.B). Bending can also be minimised by tension chording. Besides reducing the bending, it also replaces the actuation, leading to a bioinspired system. This option is illustrated in Figure 1.III.C). In Figure 1.III.C), the combination of both principles is shown. Compared to the structure in Figure 1.III.B), the mass in Figure 1.III.C) is lower. Furthermore, the beam in Figure 1.III.C) can be moved by the tension chords.

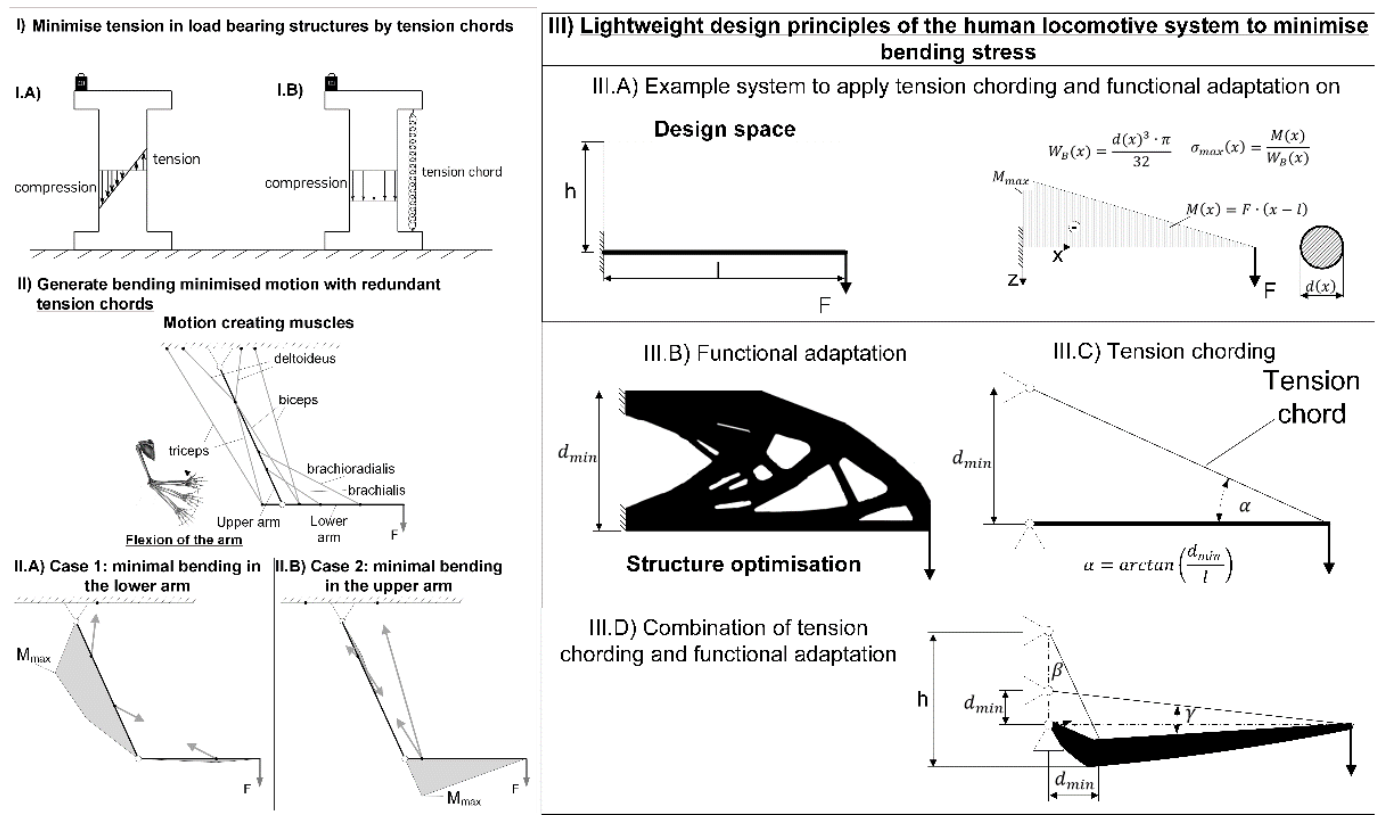

Figure 1: Basic functions of the tension chording principle (left) and the effects of the principles on the shape of a load-bearing structure (right). Adapted from Bartz et al. (2017), Bartz et al. (2018a) and Uttich et al. (2017)

To conclude, tension chording in technical applications is currently synonymous with using chords as actuators. In some systems, chords are added as extra elements to a system to reduce bending (Möhl 2003; Klug et al., 2005). But the tension chording principle, as described above, goes beyond that. The main innovation in tension chording is the functional integration of generating movement and reducing bending. That is, using redundant actuators to control the bending in structural components while generating motion at the same time by replacing the actuation principle. Thereby, no extra elements are added. To the authors' best knowledge, there are no industrial systems using this combination of redundant actuators with a bending minimising activation algorithm. This is also true 
for the interplay of tension chording, functional adaptation and motion control. In the following sections, the current state of the transfer of the above-mentioned interplay of the lightweight design principles in a bioinspired approach for technical systems is discussed.

\section{MODELS OF INTERACTION OF LIGHTWEIGHT DESIGN PRINCIPLES}

In the previous sections, the lightweight design principles of the human locomotive system are introduced. The positive effect of the interplay of tension chording and functional adaptation in the human locomotive system is illustrated with an example introduced by Pauwels (1965). Additionally, technical equivalents and their areas of application are named. In this section, the aforementioned bioinspired approach for technical design is discussed. Therefore, a model of the interaction of the lightweight design principles in the musculoskeletal system created by the authors, on which the approach is based, is presented first in order to be able to give a perspective on modifications with the help of this information. This model represents the interplay of design principles following lightweight design principles as found in the human locomotive system, containing the interplay of tension chording, motion control and functional adaptation. For the transfer of the interplay of the principles to technology, this representation is adequate. Then, the workflow derived from this model to use the interplay in the design process of a product is discussed.

\subsection{A model to describe the interplay of lightweight design principles in the human locomotive system}

To investigate potential positive effects of the interplay of principles in technical systems, the first step is to understand their interplay in the human locomotive system. To model the interplay, an analogy between the human locomotive system and a mechatronic system is drawn. The central nervous system (CNS), i.e. the brain and all the nerves connecting the brain with receptors like pain receptors, is like a microprocessor board. Muscles are actuators which need to receive signals for activation, and bones are the load-bearing structures. The model for the interplay of the principles is shown in Figure 2.

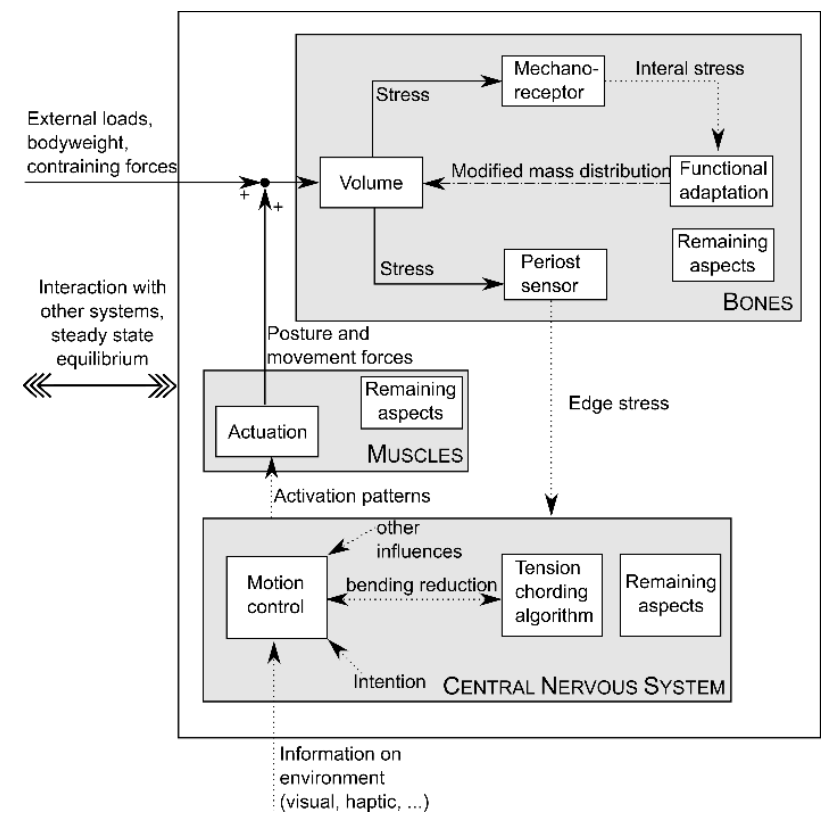

Figure 2: Model of the interplay of tension chording, functional adaptation and motion control in the human locomotive system. Adapted from (Uttich et al., 2018)

To simplify, only aspects that are assumed to be relevant for the interplay are included. For example, synthetic functions of the bone like producing blood cells or the control of facial expression in the CNS are excluded. Energy flows are displayed by arrows with straight lines, arrows with dotted lines show information flows and dot-line-arrows indicate material flows. Additionally, assumptions were made for this model, as some causalities are not yet investigated enough, like the trigger for the tension chording algorithm. Still, the model seems reasonable to describe the interplay of the principles and provides enough insights for the transfer of them. 
Several influences on the motion control are displayed in the model. One of the most important influences is the intention of the human being. It is also shown that bending reduction connects motion control with tension chording. Tension chording and motion control are assumed to be part of the CNS. Both algorithms are mainly located in the brain. Yet, some parts of them could be reflexes, which are executed in the spinal cord. The CNS generates activation patterns of the actors. Activation pattern is a term introduced to describe the pattern in which the muscles are activated to generate motion. This includes which muscles are activated and to what extent.

The forces generated by the muscles can either be posture forces, i.e. forces to keep a certain position, or movement forces. These forces are applied on the bone as well as external loads, bodyweight and constraining forces, i.e. forces applied by ligaments, joints or from other origins on the bone. Combined with the volume of the bone, stress can be measured. While internal stresses are measured by mechanoreceptors and provide the basis for functional adaptation (Frost 2003; Ruff et al., 2006), stress on the outer shell of the bone is measured by periost sensors. It is assumed, that these edge stresses are used for tension chording. This assumption is based on the fact that the information of the sensors are transferred to the CNS (Benninghoff et al., 2002). With this model, a basic understanding of the interplay is obtained. This is taken as a basis to transfer the interplay of the principles and described in the next section.

\subsection{A model of the bioinspired approach for the technical realisation of the interplay of the lightweight design principles}

Systems in nature are in steady state equilibrium with other systems, i.e. they are open systems which adapt to changing conditions (Hill 1997). A change of one parameter can lead to changed equilibrium points in several other systems. One example for this is functional adaptation. The mass of a bone is adapted to the loads it must endure (Ruff et al., 2006). In contrast to the human body, major changes to the structure of technical systems are not possible during its utilisation without replacing the structure by another. This is only possible in the design phase. Yet, tension chording is partly and motion control is completely realised by microcontrollers, and they are adaptable. It becomes obvious, that functional adaptation can only be considered in the design phase, while tension chording and motion control are relevant during design and utilisation. To cover all lightweight design principles, the transfer of their interplay will be limited to the design phase first.

Finding an optimal solution for all desired use cases during operation is the main task in the design process. This causes variations of parameters in all lightweight design principles for given boundary conditions until an equilibrium is found. While this happens simultaneously in the human locomotive system, simultaneity cannot be realised in a technical approach. For this reason, a serial approach with convergence criteria is chosen for the design process. A simplified model of the technical approach introduced by Bartz et al. (2018b) is shown in Figure 3. The human extremities, where the lightweight design principles take effect, can abstractly be described as kinematic chains. Therefore, this workflow is developed for a kinematic chain representing a simple technical system kinematically comparable with the human body. The workflow is implemented by using the computer aided design tool Solidworks, the mathematics tool MatLab and the finite element analysis tool Ansys Workbench. The serial approach allows the different algorithms to use each other's outputs. To consider the parallel operation of principles, optimisation algorithms are introduced for the workflow. Each algorithm converges to an optimum for the given inputs then used by another algorithm until mass is unchanged.

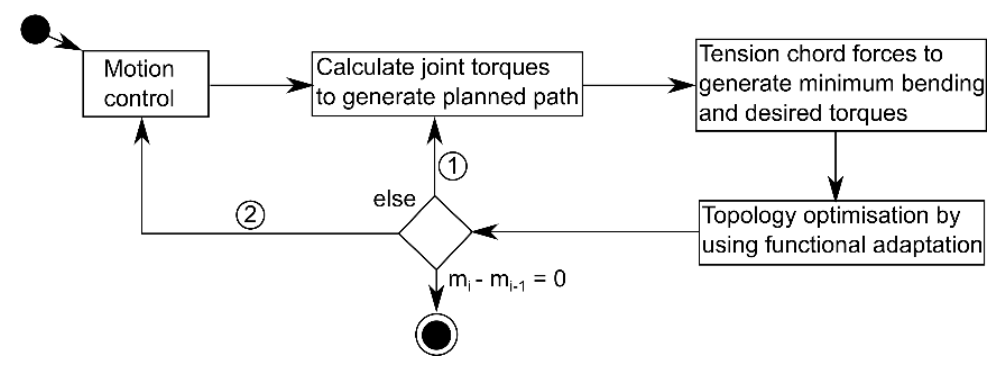

Figure 3: Simplified and extended representation of the workflow presented by Bartz et al. (2018b) for the design of a kinematic chains 
There are two different possibilities marked in the workflow. Option (1) is to calculate the desired motion once and find an equilibrium for changing tension chord forces and topology. Option (2) and realised by Bartz et al. (2018b), is to recalculate the path each time the topology and tension chord forces are changed. The resulting structure has been realised as a physical prototype.

\section{INTEGRATION OF THE AVAILABLE INSIGHTS INTO THE PROCESS AND CONTEXT OF ENGINEERING DESIGN AND APPLICATION}

The described bioinspired approach presents a first result as an intermediate step on the way to the transfer or application of the lightweight design principles of the human locomotive system to technical applications. However, the approach is abstract and it leaves many areas and questions open. At present, the bioinspired approach provides more of a calculation workflow than further assistance for the designer in a product development process. The approach does not provide any assistance in concept development, nor does it restrict itself to specific systems and does not take application-specific requirements into account. Therefore, in subsection 4.1, the bioinspired approach is first sorted into the classical design process after Pahl/Beitz (Feldhusen and Grote 2013) with the aim of looking at which problems exist and which tasks still need to be investigated. With the results of this classification, possible steps for further work are discussed in subsection 4.2. On the one hand, it is a matter of how further fields of application can be developed and on the other hand, how a detailed application-specific research of new bioinspired processes can look like for exemplarily selected applications.

\subsection{Classification of the bioinspired approach into the classical design process}

When the aforementioned calculation workflow of the bioinspired approach is used, the main output is the shape of the load-bearing structure, optimised for the use of tension chording and bending minimised motion control. To illustrate the needed inputs and the essential outputs of the calculation workflow, a black box model of the workflow is introduced in Figure 4. This is used to integrate the workflow into the development process.

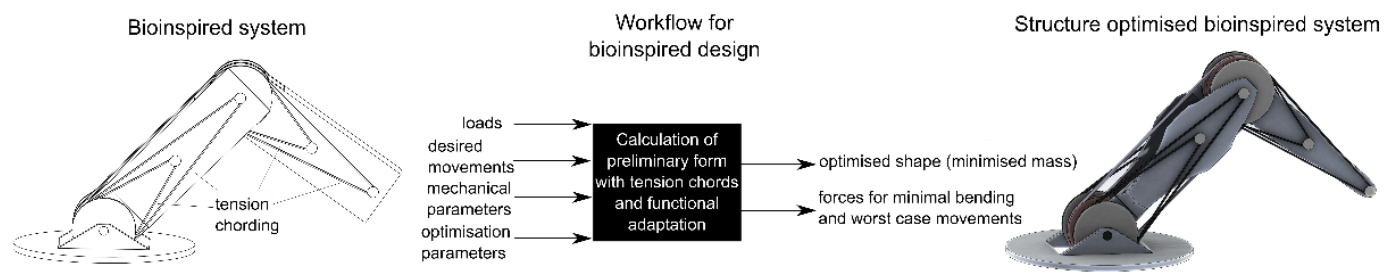

Figure 4: Black box with input and output parameters for the calculation workflow. As an example, an illustration of a bioinspired system as an input and the optimised structure of the bioinspired system as an output is added.

The term loads includes all external and internal loads not affected by the tension chords. Depending on the workflow chosen from Figure 3, desired movement is either a set of starting and ending points for workflow (2), or joint angles over time for workflow (1). Mechanical parameters include material parameters, especially Young's modulus, and measures like lever arms, degrees of freedom, movement space, locations and number of tension chords, and insertion points of other loads. Optimisation parameters include the design space, maximum accelerations for the motion control, maximum actuation force values, precision for convergence check and available energy.

Taking the product development process introduced by Pahl/Beitz as a reference, the previously mentioned insights are added to the different phases. The design process of a product is separated into four phases. The first phase is called planning and task clarification. Its result is a requirements list. In the second phase, a principle solution is being developed. This phase is called conceptual design phase. During phase three, the layout of the product is specified. This includes the design of a preliminary and definitive layout of the construction structure. The last phase is called detailed design phase. In every phase, iterations to prior phases may become necessary. As previously mentioned, the calculation workflow for bioinspired design is used to obtain a preliminary form design for the loadbearing structure. This takes place during the embodiment design phase.

Concluding from these remarks, the calculation workflow is the first step in attaining a preliminary layout, when a principle solution is given. As a result of the execution of the workflow and the 
subsequent evaluation of the layout against technical and economic criteria, changes in the concept and requirements may become necessary. Concerning the concept, these changes can affect e.g. the number of tension chords and the location of their attachment to the load-bearing structure. Additionally, the form of the available design space or other interfaces with the product concept or requirements may require changes. Then, the calculation workflow is executed with the adapted concept and requirements and again evaluated in the aftermath. This process is repeated until all criteria are met.

\subsection{Deriving fields of application and further studies for specific applications}

To get the bioinspired lightweight design approach ready to be used in applications, the authors aim for deriving a methodology to support product developers in applying the approach to subsystems and integrating the bioinspired subsystems into the system. The classification of the bioinspired approach in the process of designing of Pahl/Beitz shows that the workflow cannot be used yet for this aim. Furthermore, only one academic example on this bioinspired approach is available in form of an articulated arm robot. The boundary conditions for this example are very specific, so that it could not be used in real-world applications. In order to derive the desired methodology, more examples are needed under various boundary conditions and thus different requirements. In these examples, system integration needs to be a relevant problem of product development.

The first idea to obtain new examples is to apply the interplay of the biological lightweight design principles on other systems kinematically similar to the human locomotive system, e.g. excavators, humanoid or industrial robots. Load-bearing structures of these systems are dimensioned for various movement paths (VMP). But in the current bioinspired approach, dimensioning is done for a single movement path (SMP). The reason for this is, that for various movement paths, a different structure on the topology optimisation would be optimal through different load cases. However, there is still no concept how to ensure that a beneficial load or stress distribution can be set for VMP without constantly determining a new geometry. The human locomotive system is designed for VMP. Therefore, it is possible that this lightweight design principle can also be used for VMP systems. In the biological case a constant variation of the geometry is prevented by the fact that the movement forces (in this case applied by muscles) are controlled in a way that always leads to similar stress distributions in the structure. In order to technologically implement this control behaviour, the previously described bioinspired approach must be extended to include components of control engineering and sensor technology. Thus, transferring the biological concept to VMP systems would require additional in-depth research on the concept.

Another idea is to find new areas of application that are better suited to the unmodified bioinspired approach. In this research field, the design approach is used on a wide variety of subsystems to derive conclusions on system integration. Ideally, the design approach could be used without any changes for the chosen applications. The bioinspired subsystems should be part of complex systems in which the integration of the subsystem into the system is an issue. This would require research on the integration of the bioinspired subsystems into the system.

To obtain new fields of application, an approach inspired by step four of the methodology "from biology to an application" of (Cohen and Reich 2016) is used. First, the fulfilled function and structure of the current solution are abstracted. Then, systems are sought-after for which the abstract description is applicable. The result of this approach is the content of Figure 5.
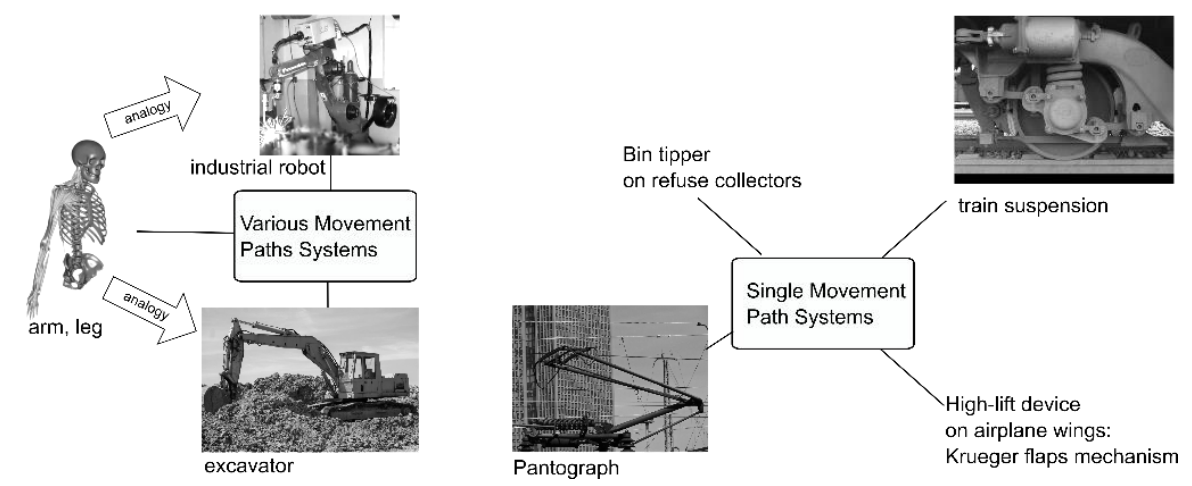

Figure 5: Examples for different types of systems for which the workflow might be applied. All pictures used are released in public domain. 
As the concept is based on lightweight design principles of the human locomotive system, a human is displayed on the bottom. While the structure of the lightweight design subsystems can be described as open kinematic chains, the main function is to move an item on paths. Examples for technical systems that match both function and structure are robots and excavators. These are VMP systems. Also, the subsystem affected by the bioinspired design approach performs the main function of the system.

In more abstract terms, the function is to transmit and transform force and movement. By describing the structure more abstractly as a kinematic chain, closed kinematic chains are included in the search for areas of application. Linkages match both the function and the structure. One example for a linkage, Watt's linkage, is displayed in use as a train suspension in Figure 5. Other examples, in which linkages are used, are Pantographs of rail cars and bin tipper on refuse collectors. Another example is the Krueger flaps mechanism to extend and retract high-lift devices of airplanes. All examples are SMP subsystems and kinematic chains, which matches the developed design approach. As these subsystems are integrated in more complex systems, research on system integration of the bioinspired parts would be possible. However, for two reasons, the design approach cannot be used for these systems yet. The first reason is, that some of the systems are closed kinematic chains, for which the usability of the design approach has not yet been proven. The second reason is, that a concept for load distribution and actuation for complex movement paths is missing. Additionally, it is unclear whether the bioinspired subsystem should replace the whole existing subsystem or if only the actuation of the subsystem should be replaced by a bioinspired actuation.

In conclusion, both directions for further research fulfil the aim to bring the concept closer to application. Still, the existing lightweight design approach cannot be used in any of the directions without further research on the lightweight design concept. Concepts to realise the calculated force distributions in service are needed. In the next section, it is discussed whether in-depth research on the design approach or research on system integration is more beneficial to the development of the methodology.

\section{CONCLUSION AND DISCUSSION OF RESEARCH GAPS}

Allocating the existing lightweight design approach in a process of design has shown that the design approach is not ready to be used by product developers yet. A methodology is needed which supports the product developer with the application of the lightweight design approach on subsystems and the integration of the bioinspired subsystems into the system. In order to transfer the design approach into a methodology, the design approach must be applied on a wide variety of subsystems to enable research on the integration of the bioinspired subsystems. But to apply the design approach on the subsystems, research on the design approach is needed. In VMP systems, the bioinspired subsystem can often be changed without major impacts on other subsystems. Often, this is not the case for SMP systems. The complexity of system integration in SMP systems could hinder in-depth research on the design approach.

Investigating yet unanswered questions for the design approach in VMP systems could lead to an understanding of the design approach from which the research on system integration of the bioinspired subsystem benefits. Therefore, in-depth research on the design approach is chosen as the direction of further research.

In order to apply the design approach to e.g. a specific robot system, it must include dimensioning for VMP. This requires an adaptation of the load case selection process and eventually also of the convergence criteria of the workflow. Additionally, the load distribution algorithm must be adapted to consider several load cases and find the optimum load distribution for all of them. The existing algorithm calculates the distribution for each load case individually, which can lead to explicitly different load distributions and consequently high masses. Another important aspect to investigate are concepts to ensure the load distribution in service. These concepts can range between mechanical and mechatronic concepts. The latter would include load distribution steering and control concepts. The authors assume that for VMP systems and complex movements of SMP systems the use of a steering approach is inevitable. 


\section{REFERENCES}

Ananthanarayanan, A., Azadi, M. and Kim, S. (2012), "Towards a bio-inspired leg design for high-speed running", In: Bioinspiration \& Biomimetics, Vol. 7 No. 4, p. 46005. https://dx.doi/org/10.1088/17483182/7/4/046005.

Bartz, M., Gößling, R., Schafran, T. and Bender, B. (2017), “Entwicklung eines bioinspirierten Gelenkarmroboters mithilfe der Kopplung von Mehrkörpersimulation und Topologieoptimierung. Koblenz: 35", CADFEM Ansys Simulation Conference.

Bartz, M., Brand, H. and Bender, B. (2018a), "Examing lightweight design potential of the human musculoskeletal system by using the example of an articulated arm robot", In: Book of Abstracts: 1 . Symposium for Lightweight Design in Product Development. 13. - 15. June 2018. Zurich.

Bartz, M., Gößling, R., Remus, R. and Bender, B. (2018b), "Development of a bioinspired approach for the design of kinematic chains", In: Proceedings of the DESIGN 2018, 15th International Design Conference Dubrovnik, Croatia, 21/05/2018 - 24/05/2018, pp. 975-984. https://dx.doi/org/10.21278/idc.2018.0330.

Bartz, M. (2019), Transfer des Muskuloskelettalen Leichtbaus auf Offene Kinematische Ketten. Verlag Dr. Hut, München. ISBN: 978-3-8439-3935-5.

Benninghoff, A., Drenckhahn, D. and Zenker, W. (2002), Anatomie 1. München, Jena, Urban \& Fischer, Elsevier, Amsterdam.

Cohen, Y. H. and Reich, Y. (2016), Biomimetic Design Method for Innovation and Sustainability. Springer.

El Khoury, A., Lamiraux, F. and Taix, M. (2013), “Optimal Motion Planning for Humanoid Robots”, IEEE International Conference on Robotics and Automation (ICRA), May 2013, Karlsruhe, Germany, 2013.

Feldhusen, J. and Grote (2013), Pahl/Beitz Konstruktionslehre: Methoden und Anwendung erfolgreicher Produktentwicklung. Springer Vieweg, Berlin, Heidelberg. ISBN 978-3-642-29568-3.

Feyerabend, F. (1991), Wertanalyse Gewicht: Methodische Gewichtsreduzierung am Beispiel von Industrierobotern. Fortschritt-Berichte der VDI Zeitschriften, VDI (Hrsg.), (Reihe 1, Nr. 201), 1991.

Fratzl-Zelman, N., Misof, B. M. and Roschger, P. (2011), "Das knochenmaterial: Ein nano-komposit aus mineral und kollagen", Journal für Mineralstoffwechsel, 2011 Vol. 18 No. 3, pp. 110-117, 2011.

Frost, H. M. (2003), “Bone's mechanostat: A 2003 update”, The Anatomical Record. Part A, Discoveries in Molecular, Cellular, and Evolutionary Biology, 2003 Vol. 275 No. 2, pp. 1081-1101, 2003.

Gößling, R., Herzog, M., Witzel, U. and Bender, B. (2014), "Compensation of bending moments as a natureinspired design principle?", In: Design 2014: Proceedings of the 13th International Design Conference, May 19-22, 2014, Dubrovnik - Croatia, pp. 193-200.

Hill, B. (1997), Innovationsquelle Natur. Naturorientierte Innovationsstrategie für Entwickler, Konstrukteure und Designer. Shaker Verlag, Aachen.

Klug, S., Möhl, B., Stryk, O. V. and Barth, O. (2005), "Design and Application of a 3 DOF Bionic Robot Arm", AMAM 2005, Illmenau, Germany, September 25-30, 2005, 2005.

Mattheck, C. (1997), Design in der Natur. Rombach GmbH + Co Verlagshaus KG, Freiburg, 1997.

Möhl, B. (2003), “A Composite Drive with Separate Control of Force and Position”, In: Proc.of the 11th International Conference on Advanced Robotics 2003 in Coimbra (ICAR 2003), pp. 1606-1610, 2003.

Mombaur, K., Koch, K. H. and Felis, M. L. (2014), "Model-based optimization for robotics. Journal of the Robotics Society of Japan", Special Issue on Optimization Used in Robotics Research, Vol. 32, 2014.

Nachtigall, W. (2010), Bionik als Wissenschaft. Springer, Heidelberg, Dordrecht, London, New York. 2010.

Pauwels, F. (1965), Gesammelte Abhandlungen zur Funktionellen Anatomie des Bewegungsapparates. SpringerVerlag, Berlin, Heidelberg, New York. 1965.

Ruff, C., Holt, B. and Trinkaus, E. (2006), “Who's afraid of the big bad Wolff?: Wolff's law and bone functional adaptation”, American Journal of Physical Anthropology, Vol. 2006 No. 129, pp. 484-498, 2006.

Uttich, E., Gößling, R., Bartz, M. and Bender, B. (2017), "Inversdynamische Berechnungen der Muskelkräfte am Glenohumeralgelenk unter der Prämisse der Biegeminimierung”, In: 35. CADFEM ANSYS Simulation Conference: die Fachkonferenz zur Numerischen Simulation in der Produktentwicklung, 15.-17. November 2017, Koblenz.

Uttich, E., Bartz, M. and Bender, B. (2018), "Review of the mechanisms of action in the musculoskeletal system as a basis for new simulation models", 8th World Congress of Biomechanics, 8-12 July 2018, Dublin, Ireland, 2018.

VDI 6224-3 (2017), Bionik - Bionische Strukturoptimierung im Rahmen eines ganzheitlichen Produktentstehungsprozesses. Beuth-Verlag, Berlin, 2017.

Weber, W. (2017), Industrieroboter. Carl Hanser Verlag, München.

Witte, H., Fischer, M.S., Schilling, N., Ilg, W., Dillmann, R., Eckert, M. and Wittenburg, J. (2000), Konstruktion vierbeiniger Laufmaschinen anhand biologischer Vorbilder. Konstruktion 9-2000, pp. 46-50, 2000. 